\title{
Publisher's Note: Spinning test body orbiting around a Schwarzschild black hole: Circular dynamics and gravitational-wave fluxes [Phys. Rev. D 94, 104010 (2016)]
}

Enno Harms, Georgios Lukes-Gerakopoulos@, Sebastiano Bernuzzi, and Alessandro Nagar

(Q) (Received 6 December 2019; published 17 December 2019)

DOI: 10.1103/PhysRevD.100.129902

This paper was published online on 4 November 2019 with errors in Eqs. (21), (40), (43), and (49) and related text. The equations and related text have been corrected as of 13 December 2019. The equations and related text are incorrect in the printed version of the journal. 